\title{
Exploratory Radiomics in Computed Tomography Perfusion of Prostate Cancer
}

\author{
STEPHANIE TANADINI-LANG ${ }^{1}$, MARTA BOGOWICZ ${ }^{1}$, PATRICK VEIT-HAIBACH ${ }^{2,3}$, MARTIN HUELLNER $^{2}$, \\ CHANTAL PAULI $^{4}$, VYOMA SHUKLA ${ }^{1}$, MATTHIAS GUCKENBERGER $^{1}$ and OLIVER RIESTERER $^{1}$ \\ ${ }^{1}$ Department of Radiation Oncology, University Hospital Zurich, University of Zurich, Zurich, Switzerland; \\ ${ }^{2}$ Department of Nuclear Medicine, University Hospital Zurich, University of Zurich, Zurich, Switzerland; \\ ${ }^{3}$ Institute of Diagnostic and Interventional Radiology, \\ University Hospital Zurich, University of Zurich, Zurich, Switzerland; \\ ${ }^{4}$ Institute of Surgical Pathology, University Hospital Zurich, University of Zurich, Zurich, Switzerland
}

\begin{abstract}
Background/Aim: An evaluation if radiomic features of CT perfusion (CTP) can predict tumor grade and aggressiveness in prostate cancer was performed. Materials and Methods: Forty-seven patients had biopsy-confirmed prostate cancer, and received a CTP. Blood volume (BV), blood flow (BF) and mean transit time (MTT) maps were derived and 1,701 radiomic features were determined per patient. Regression models were built to estimate postsurgical Gleason score (GS), microvessel density (MVD) and distinguish between the different risk groups. Results: Six out of the 47 patients had to be excluded from further analysis. $A$ weak relationship between postsurgical GS and one radiomic parameter was found $\left(R^{2}=0.21, p=0.01\right)$. The same parameter combined with MTT inter-quartile range was prognostic for the risk group categorisation $(A U C=0.81)$. Two different radiomic parameters were able to distinguish between low-intermediate risk and high-intermediate risk (AUC=0.77). Four parameters correlated with MVD $(R 2=0.53, p<0.02)$. Conclusion: This exploratory study shows the potential of radiomics to classify prostate cancer.
\end{abstract}

Prostate cancer is the most common malignant tumor in men in Europe (1). Its incidence is increasing since the late 1970s and it is expected to further increase in the future.

Tumor grading, i.e. the Gleason score, together with PSA serum concentration and $\mathrm{T}$ stage is important to assign risk

This article is freely accessible online.

Correspondence to: Stephanie Tanadini-Lang, Department of Radiation Oncology, Rämistrasse 100, 8091 Zürich, Switzerland Tel: +41 442554238, e-mail: stephanie.tanadini-lang@usz.ch

Key Words: Radiomics, computed tomography perfusion, prostate cancer. groups and offer an adequate therapy to the patient. The current standard for tumor grading is histopathological assessment of core-needle biopsy probes. The limitation of this approach is that only a limited number of biopsies can be taken, which leads to the risk of missing the tumor. Heidegger et al. showed that pre-surgical biopsy-derived tumor grading differed significantly from the grading after radical prostatectomy $(2,3)$.

Besides tumor grade, the microvessel density (MVD) is a histopathologic surrogate for angiogenesis or aggressiveness in many tumors $(4,5)$. In a retrospective analysis of tissue microarrays containing several thousand prostate cancer biopsies, a high MVD correlated with biochemical failure after prostatectomy (5). However, the assessment of this parameter in biopsies is so far not feasible in clinical practice. Therefore, there is a high interest for a non-invasive assessment of the MVD with functional imaging.

Recently, multiparametric magnetic resonance imaging (MRI) has become a standard for prostate cancer diagnosis and prediction of tumor aggressiveness (6-8). However, many patients have contraindications to MRI, such as claustrophobia, metallic foreign bodies, or pacemakers. Another approach is computed tomography perfusion (CTP) that provides blood volume (BV), blood flow (BF) and mean transit time (MTT) values as a surrogate for angiogenesis and might therefore be a promising tool for the grading of prostate tumors, especially in patients with contraindications to MRI. Two studies found an association between tumor grade and mean perfusion parameters $(9,10)$. Huellner et al. showed that the mean BF distinguished better between high grade and intermediate grade tumors than the presurgical Gleason score (GS) (10).

Radiomics is a technique to extract quantitative features automatically from medical images. In some studies, up to 1000 features per image were extracted, which enables more detailed tumor quantification than clinically used imaging metrics. Such features were shown to be prognostic for treatment outcome and associated with biological parameters 
(11-13). This makes CTP radiomics a potential powerful tool for the assessment of tumor grade and stage in prostate cancer.

The aim of this study was to evaluate if radiomic features of CTPs can predict tumor grade and aggressiveness in prostate cancer. In 47 patients, a CTP scan was performed before surgery and the association between BF, BV and MTT radiomics parameters and GS, risk group (RG) and MVD was investigated.

\section{Materials and Methods}

Patient population and image acquisition. This retrospective analysis was performed on datasets of 47 patients collected in prospectively and approved by the local ethical commission. Informed consent was obtained from all patients. Patients were assigned to the following risk groups based on GS: low risk (GS $\leq 6)$, intermediate risk (GS 7) or high risk (GS 8-10). The intermediate risk group was further subdivided into lowintermediate (GS 3+4) or high-intermediate (GS 4+3) (14).

All patients had biopsy confirmed prostate cancer, and underwent a CTP scan prior to radical prostatectomy. CTP imaging was performed on a Somatom Definition Flash scanner (Siemens Healthcare, Erlangen, Germany) using the scanning protocol previously published by Hüllner et al. (10) $(100 \mathrm{kV}, 100 \mathrm{mAs}$, time between two scans $=1 \mathrm{~s}$, slice thickness $=3 \mathrm{~mm}$ ). Iodinated contrast medium (40 ml of Ultravist ${ }^{\circledR} 370$, Bayer Schering Healthcare, Berlin, Germany) was injected at a flow rate of $4.5 \mathrm{ml} / \mathrm{s}$, and after a delay of 3 to 5 seconds, the acquisition of CTP scans was started. Images were reconstructed using filtered back projection with an inplane resolution between 0.5 and $1 \mathrm{~mm}$

This prospective study was approved by the institutional review board and by the cantonal ethics committee. All patients provided informed signed consent before the examinations.

Perfusion maps calculation. First, the motion of the prostate during the CT series acquisition was determined. If the motion was larger than $3 \mathrm{~mm}$, the patient was excluded from further analysis. Then the resolution and slice thickness of all CT scans was adjusted to the maximum slice thickness used $(3 \mathrm{~mm})$. Based on these CTs scans the three perfusion maps, blood volume (BV), blood flow (BF) and mean transit time (MTT) were calculated using an in-house developed software, which is based on the singular value decomposition method $(15,16)$. All maps were normalized to the perfusion values inside the external iliac artery. To exclude bone and air from the maps, only tissue in the Hounsfield unit range from -100 to 180 was used.

The prostate without seminal vesicles was delineated on one of the CT image batches of the perfusion series and perfusion parameters were only calculated inside these contours.

Radiomics analysis. In total, 1,701 radiomic features per patient were determined in the three perfusion maps (567 parameters per map). The following 63 features were calculated in the nontransformed maps and their wavelet transforms (8 sub-bands) (12):

- the histogram of intensities (HI) parameters $(n=17)$

- the Gray Level Co-occurrence Matrix (GLCM) parameters $(n=26)$

- the Neighborhood Gray Tone Difference Matrix (NGTDM) parameters $(n=5)$

- the Gray Level Size Zone Matrix (GLSZM) parameters $(n=14)$

- the fractal dimension $(\mathrm{n}=1)$
The analysis was performed using an in-house developed software solution implemented in Python programming language (v. 2.7). The wavelet decomposition was performed using PyWavelets library and the 'Coif1' wavelet (12). Histogram-based parameters were calculated on the original image intensities, whereas for texture quantification perfusion maps and their wavelet transforms were discretized into 64 bins, using patient-specific minimum and maximum intensity. The full list of calculated radiomic features will be provided by the corresponding author upon request.

Histopathological analysis. The GS was assessed according to international guidelines (17). The mean MVD in the tumor was determined according to immuno-histochemical cluster of differentiation molecule 34 (CD34) staining as previously published by Huellner et al. (10).

Statistical analysis. To reduce the dimensionality of the dataset, a principal component analysis (PCA) was performed. Horn's parallel analysis was used to determine the number of components to retain in PCA. All radiomic parameters were investigated with regard to the retained principal components using Spearman correlation. For each principal component, the radiomic parameter with the largest absolute correlation coefficient was taken to represent the group of parameters associated with this principal component. Multivariable regression models were learned with these radiomic parameters to estimate postsurgical GS and MVD. Additionally, a model to predict the post-surgical GS was learned using the radiomic parameters and the biopsy-derived GS.

To distinguish between low (GS<7) and intermediate (GS=7) risk groups and between intermediate and high $(\mathrm{GS}>7)$ risk groups, logistic regression models were learned. All patients in the intermediate risk group were divided into two groups, patients with a GS 3+4 (low-intermediate risk) and a GS 4+3 (high-intermediate). A logistic model was learned to distinguish these two subgroups. For all models 10 -fold cross validation was used to estimate the prediction accuracy. The area under the curve (AUC) was calculated to show sensitivity and specificity of these models.

\section{Results}

Six out of the 47 patients had to be excluded from the analysis because the motion during the CT series was exceeding $3 \mathrm{~mm}$. The most common GS was 7, both at biopsy and at wholemount histopathology after surgery (Table I). In 13 patients $(28 \%)$, there was a change of the grading from biopsy results to postsurgical histopathology, with an upgrading in 10 patients. There was a weak correlation between biopsy-derived GS and postsurgical GS $\left(\mathrm{R}^{2}=0.22, p=0.002\right)$. The microvessel density was determined in 30 out of 41 patients, the mean value was $148.3 \pm 52.0$ microvessels $/ \mathrm{mm}^{2}$ (Table I). The median time interval between CTP scan and biopsy was 16 days, whereas the median time interval between CTP scan and surgery was 69 days.

Radiomic parameters selection. A total of 1701 radiomic features were calculated, 87 of them were excluded because they had zero variation among the patients. The remaining 1,614 parameters were grouped into 10 groups using the 
Table I. Patient characteristics.

\begin{tabular}{lc}
\hline Biopsy-derived Gleason score & $\mathrm{N}$ \\
6 & 7 \\
7 & 28 \\
8 & 4 \\
9 & 2 \\
Postsurgical Gleason score & $\mathrm{N}$ \\
6 & 1 \\
7 & 32 \\
8 & 5 \\
9 & 3 \\
Microvessel density: & $148.3 \pm 2.0$ \\
Mean \pm SD (microvessels/mm ${ }^{2}$ ), $\mathrm{n}=30$ & \\
Time between CTP and surgery & $69(10-300)$ \\
Median (range) (days) & \\
Time between biopsy and CTP & $16(0-202)$ \\
Median (range) (days) &
\end{tabular}

Table II. Ten radiomic parameters were determined by the principal component analysis and considered in the multivariable regression model. Four parameters had a significant influence on the MVD, two on the prediction of the risk group, two to distinguish the intermediate risk group (IRG) into low-intermediate and high-intermediate, and one parameter to predict post-surgical GS.

\begin{tabular}{lcc}
\hline $\begin{array}{l}\text { Principle } \\
\text { component }\end{array}$ & $\begin{array}{c}\text { Most correlated } \\
\text { parameter }\end{array}$ & $\begin{array}{c}\text { Significant influence } \\
\text { on model }\end{array}$ \\
\hline 1 & BV HHL coarseness & \\
2 & BV HLH root mean square & IRG \\
3 & MTT median & MVD \\
4 & BF LLH kurtosis & RG, MVD \\
5 & MTT inter-quartile range & MVD \\
6 & BV LLL difference entropy & GS, RG, MVD \\
7 & BV difference average & IRG \\
8 & BF HHL joint average & \\
9 & BF HHH fractal dimension & \\
10 & BV HLL correlation & \\
\hline
\end{tabular}

principal component analysis. For each group, the radiomic parameter that correlated best with the principal component of the group was chosen to represent the group (Table II).

Estimation of Gleason score. A multivariate regression analysis was performed including all ten radiomic parameters to predict the GS. A significant but weak relationship between postsurgical GS and one radiomic parameter was found $\left(\mathrm{R}^{2}=0.21, p=0.01\right)$ : The joint average parameter in the HHL wavelet transform of the BF map. The model could be improved by combining the radiomic parameter with the biopsy-derived GS $\left(\mathrm{R}^{2}=0.33, p=0.002\right)$.

Estimation of risk group. There was only one patient in the low-risk group, therefore no modelling could be performed

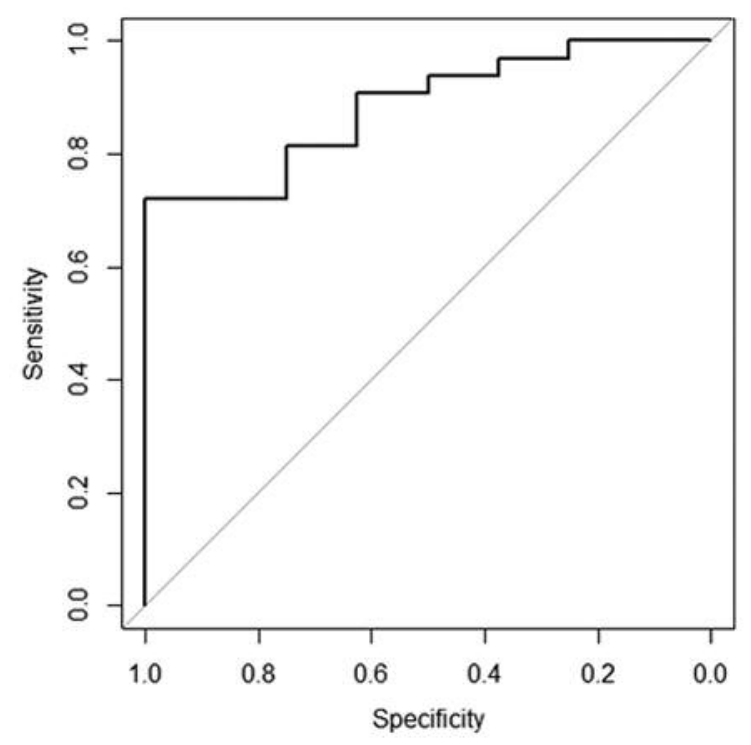

Figure 1. The receiver operating characteristics for risk group prognosis (AUC $=0.81)$.

to compare low and intermediate risk groups. A logistic regression was performed comparing intermediate and high risk groups. Two radiomic parameters had a significant contribution to the model (Table II). The AUC of this model was 0.81 (Figure 1). Both radiomic parameters had lower values in the intermediate risk group (Figure 2).

Comparison between low-intermediate risk and highintermediate risk. The patients with a postsurgical GS of 7 were subdivided into 2 groups: GS $3+4$ (19 patients) and GS 4+3 (13 patients). A logistic regression model was built to distinguish between these two groups. Two radiomic parameters significantly contributed to the model (AUC $=0.77$, Figure 3). The BV HLH inverse difference parameter was higher for the GS $3+4$ group compared to the $4+3$ group, whereas the BF $\mathrm{HHH}$ fractal dimension was smaller (Figure 4).

Correlation with MVD. Four radiomic parameters had a significant correlation with the MVD $\left(\mathrm{R}^{2}=0.53, p<0.02\right)$. The MVD was increasing with increasing interquartile range in the non-transformed MTT map and joint average in the HHL transformed BF map. The MVD was decreasing with increasing difference entropy in the LLL transform of the BV map and the kurtosis in the LLH of the BF map.

\section{Discussion}

Prostate cancer is a very heterogeneous disease. Therapeutic options include surgery, radiotherapy, hormone therapy or a combination thereof. In order to guide treatment, patients are 
Meant Transit Time, Interquartile Range

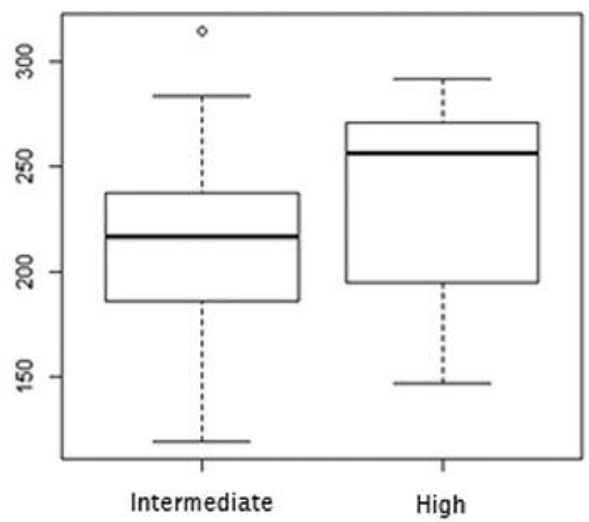

Blood Flow, HHL Joint Average

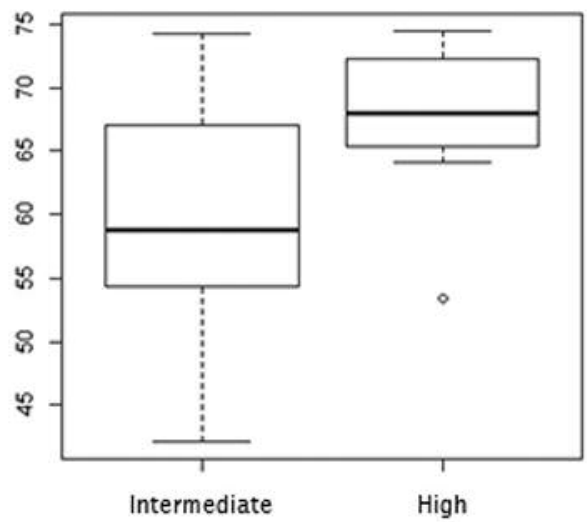

Figure 2. Boxplots for the intermediate risk and high risk group for the two significant parameters in the model.

routinely allocated to low, intermediate or high-risk groups based on tumor stage, tumor grade and PSA serum concentration. We assessed radiomics to non-invasively estimate postsurgical GS, risk group categorisation and microvessel density (MVD) based on CTP imaging. We found one radiomic parameter (BF HHL joint average) to be prognostic for the GS. This parameter and in combination with MTT inter-quartile range was prognostic for the risk group categorisation. Based on a recent publication that suggested to further divide intermediate risk prostate cancer into low-intermediate (Gleason $3+4)$ and high-intermediate (Gleason $4+3$ ) risk groups due to substantial differences in clinical outcome that require different treatment strategies (14), we found two different radiomic parameters $\mathrm{BF} \mathrm{HHH}$ fractal dimension and BV HLH root mean square) that distinguish between low-intermediated risk and high-intermediate risk. Four parameters correlated with MVD, which is an angiogenesis-related marker associated with improved prognosis.

In this study, the final postsurgical GS was incorrectly estimated by biopsy in $28 \%$ of the patients. This is in agreement with other published studies, which showed a disagreement between biopsy-derived GS and postsurgical GS between $26-71 \%(18,19)$. Radiomics in combination with the biopsy-derived GS improved the prediction accuracy.

Huellner et al. used CTP imaging to predict GS and MVD (10). They did not find a correlation between mean values of $\mathrm{BF}, \mathrm{BV}$ and MTT with MVD. Using radiomics we could build a model to predict the MVD based on 4 radiomic parameters $\left(\mathrm{R}^{2}=0.53\right)$. Tumors characterized with increased MVD had a higher MTT interquartile range and lower BF LLH kurtosis. These radiomic parameters likely correspond to a more heterogeneous blood flow, which could be

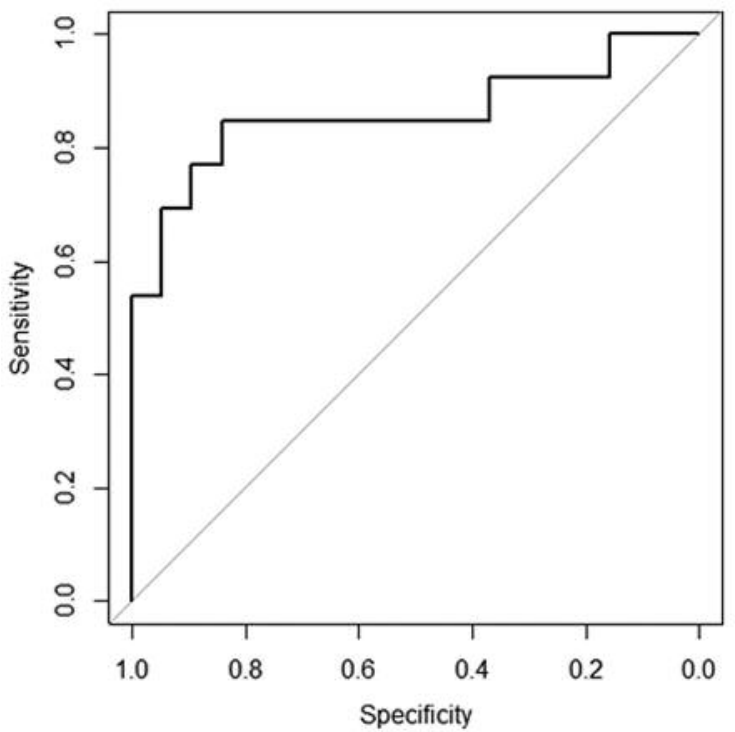

Figure 3. The receiver operating characteristics for distinguishing between low-intermediate $(G S 3+4)$ and high-intermediate $(G S 4+3)$ risk groups $(A U C=0.77)$.

explained by tortuous tumor vasculature. The biological and physiological implications of this still needs to be determined.

Our correlation model based on one radiomic parameter to predict the GS was slightly superior $\left(\mathrm{R}^{2}=0.22\right)$ to the model by Hüllner et al., which is based on the mean BV value $\left(\mathrm{R}^{2}=0.18\right)(10)$. We could additionally improve the model by combining radiomics with presurgical GS. Similar results were found for the prediction of the risk group. 

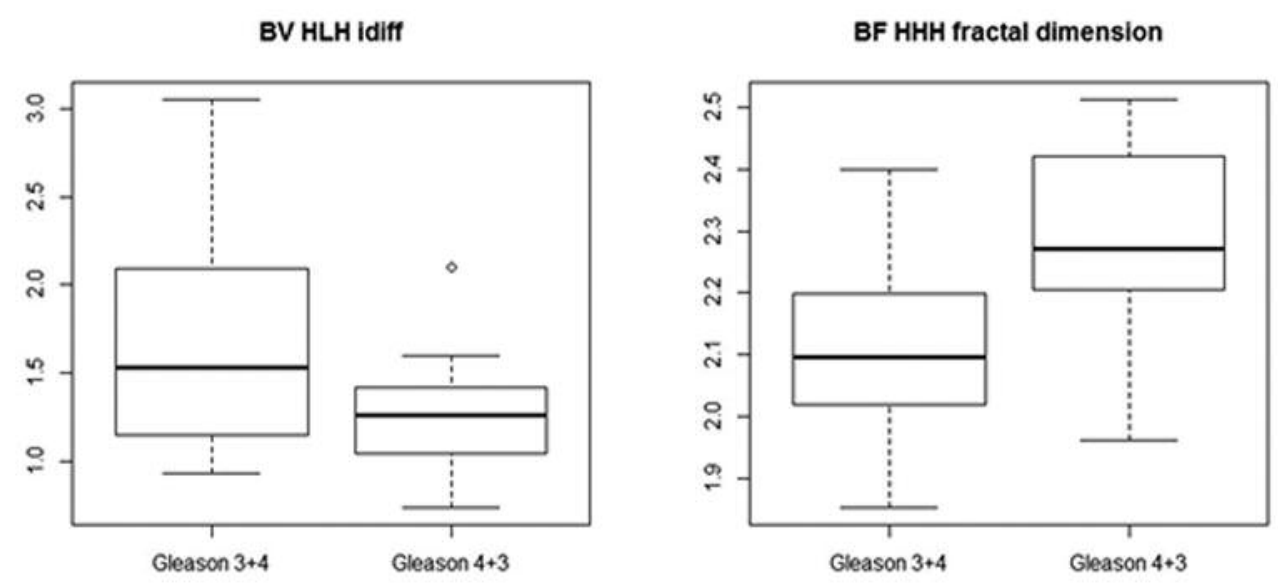

Figure 4. Boxplots for the group of patients with low-intermediate risk (3+4) and high-intermediate risk (4+3) for the two significant parameters in the model.

Several authors used radiomic analysis of multi-parametric MRI imaging for tumor grading and could show a good performance of their models to distinguish low and intermediate risk tumors $(\mathrm{AUC}=0.83-0.92)(6,7)$. The performance of the models to distinguish low-intermediate and high-intermediate GS was slightly worse (AUC $=0.59$ 0.77) $(6,8)$. Our models for CTP imaging performed similarly well (AUC $=0.77-0.81$ ) to distinguish intermediate from high risk patients and GS $3+4$ from $4+3$.

Radiomic analysis for predicting patient outcome or tumor phenotype is most often performed by delineating the tumor and performing radiomic analysis in the tumor volume itself. In our study the entire prostate was used for the analysis, because specificity and sensitivity of detecting the tumor lesion inside the prostate using computed tomography and functional imaging is not high enough (20). Additionally, often two or more lesions are present inside the prostate. We were able to show that the radiomic analysis of the entire prostate compared to an individual tumor lesion is a valid approach for the assessment of prostate cancer. This concept might be translated to other tumor entities, which would reduce the delineation work.

In our study, we calculated 3 different types of radiomic features: first order statistical parameters, texture parameters and wavelet-transform parameters. No shape parameters were calculated because not the tumor lesion itself was delineated but the entire prostate. Since the shape of the prostate does not differ much among patients, we decided not to include shape parameters.

A drawback of the study is the small number of patients included and the missing validation dataset. CT perfusion imaging is currently not standard of care for prostate cancer patients. Before applying this imaging technique to a large number of patients, its value needs to be proven on a small number of patients in clinical studies. Therefore, this exploratory study should motivate to further investigate CTP imaging for staging and grading of prostate cancer, especially for patients with contraindications to MRI. To overcome the small number of patients included in the study, we reduced the dimensionality of the dataset by performing a principal component analysis. Out of the 1,701 radiomic parameters, only the ten parameters with the best correlation to the first ten principal components were used for modelling.

\section{Conclusion}

This exploratory study showed the potential of CTP radiomics for prostate cancer classification. The analysis of a larger patient cohort in the future will allow for a more advanced radiomic parameter selection and will eventually improve the prognostic models.

\section{References}

1 Ferlay J, Steliarova-Foucher E, Lortet-Tieulent J, Rosso S, Coebergh JW, Comber H, Forman D and Bray F: Cancer incidence and mortality patterns in Europe: estimates for 40 countries in 2012. Eur J Cancer 49(6): 1374-1403, 2013.

2 Heidegger I, Skradski V, Steiner E, Klocker H, Pichler R, Pircher A, Horninger $\mathrm{W}$ and Bektic J: High Risk of undergrading and -staging in prostate cancer patients eligible for active surveillance. PLoS ONE 10(2): e0115537, 2015.

3 D'Elia C, Cerruto, MA, Cioffi A, Novella G, Cavalleri S and Artibani W: Upgrading and upstaging in prostate cancer: From prostate biopsy to radical prostatectomy. Mol Clin Oncol 2(6): 1145-1149, 2014.

4 Bunsiripaiboon P, Sornmayura P, Wilasrusmee C and Lertsithichai P: The prognostic significance of microvessel density in intrahepatic cholangiocarcinoma. J Med Assoc Thai 93(1): 66-72, 2010. 
5 Erbersdobler A, Isbarn H, Dix K, Steiner I, Schlomm T, Mirlacher M, Sauter G and Haese A: Prognostic value of microvessel density in prostate cancer: a tissue microarray study. World J Urol 28(6): 687-692, 2010.

6 Bono A, Celato N, Cova V, Salvadore, M., Chinetti S and Novario, R: Microvessel density in prostate carcinoma. Prostate Cancer Prostatic Dis 5(2): 123-127, 2001.

7 Chen YJ, Chu WC, Pu YS, Chueh SC, Shun CT and Tseng WY: Washout gradient in dynamic contrast-enhanced MRI is associated with tumor aggressiveness of prostate cancer. J Magn Reson Imaging 36(4): 912-919, 2012.

8 Vos EK, Litjens GJ, Kobus T, Hambrock T, Hulsbergen-van de Kaa CA, Barentsz JO, Huisman HJ and Scheenen TW: Assessment of prostate cancer aggressiveness using dynamic contrast-enhanced magnetic resonance imaging at $3 \mathrm{~T}$. Eur Urol 64(3): 448-455, 2013.

9 Łuczyńska E, Heinze-Paluchowska S, Blecharz P, JereczekFossa B, Petralia G, Bellomi M and Stelmach A: Correlation between $\mathrm{CT}$ perfusion and clinico-pathological features in prostate cancer: a prospective study. Med Sci Monit 21: 153$162,2015$.

10 Huellner MW, Pauli C, Mattei A, Ross S, Diebold J, Vosbeck J, Allgayer B, Strobel K nad Veit-Haibach P: Assessment of prostate cancer with dynamic contrast-enhanced computed tomography using an en bloc approach. Invest Radiol 49(9): 571-578, 2014

11 Yip SS and Aerts HJ: Applications and limitations of radiomics. Phys Med Biol 61(13): R150-166, 2016.

12 Aerts HJ, Velazquez ER, Leijenaar RT, Parmar C, Grossmann P, Carvalho S, Bussink J, Monshouwer R, Haibe-Kains B, Rietveld D, Hoebers F, Rietbergen MM, Leemans CR, Dekker A, Quackenbush J, Gillies RJ and Lambin P: Decoding tumour phenotype by noninvasive imaging using a quantitative radiomics approach. Nat Commun 5: 4006, 2014.

13 Hatt M, Tixier F, Pierce L, Kinahan PE, Le Rest CC and Visvikis D: Characterization of PET/CT images using texture analysis: the past, the present... any future? Eur J Nucl Med Mol Imaging 44(1): 151-165, 2017.
14 Zumsteg ZS and Zelefsky MJ: Short-term androgen deprivation therapy for patients with intermediate-risk prostate cancer undergoing dose-escalated radiotherapy: the standard of care? Lancet Oncol 13(6): e259-e269, 2012.

15 Ostergaard L, Weisskoff RM, Chesler DA, Gyldensted C and Rosen BR: High resolution measurement of cerebral blood flow using intravascular tracer bolus passages. Part I: mathematical approach and statistical analysis. Magn Reson Med 36(5): 715$725,1996$.

16 Nesteruk M, Lang S, Veit-Haibach P, Studer G, Stieb S, Glatz S, Hemmatazad H, Ikenberg K, Huber G, Pruschy M, Guckenberger M, Klöck S and Riesterer O: Tumor stage, tumor site and HPV dependent correlation of perfusion CT parameters and (18F)-FDG uptake in head and neck squamous cell carcinoma. Radiother Oncol 117(1): 125-131, 2015.

17 Epstein JI, Allsbrook WC Jr., Amin MB and Egevad LL, ISUP Grading Committee: The 2005 International Society of Urological Pathology (ISUP) consensus conference on Gleason grading of prostatic carcinoma. Am J Surg Pathol 29(9): 1228$1242,2005$.

18 San Francisco IF, DeWOLF WC, Rosen S, Upton M and Olumi AF: Extended prostate needle biopsy improves concordance of Gleason grading between prostate needle biopsy and radical prostatectomy. J Urol 169(1): 136-140, 2003.

19 Lattouf JB and Saad F: Gleason score on biopsy: is it reliable for predicting the final grade on pathology? BJU Int 90(7): 694698, 2002.

20 de Rooij M, Hamoen EH, Fütterer JJ, Barentsz JO and Rovers MM: Accuracy of multiparametric MRI for prostate cancer detection: a meta-analysis. AJR Am J Roentgenol 202(2): 343$351,2014$. 